\title{
Research of an Optical Device Based on a Anisotropic Epsilon-Near-Zero Metamaterial
}

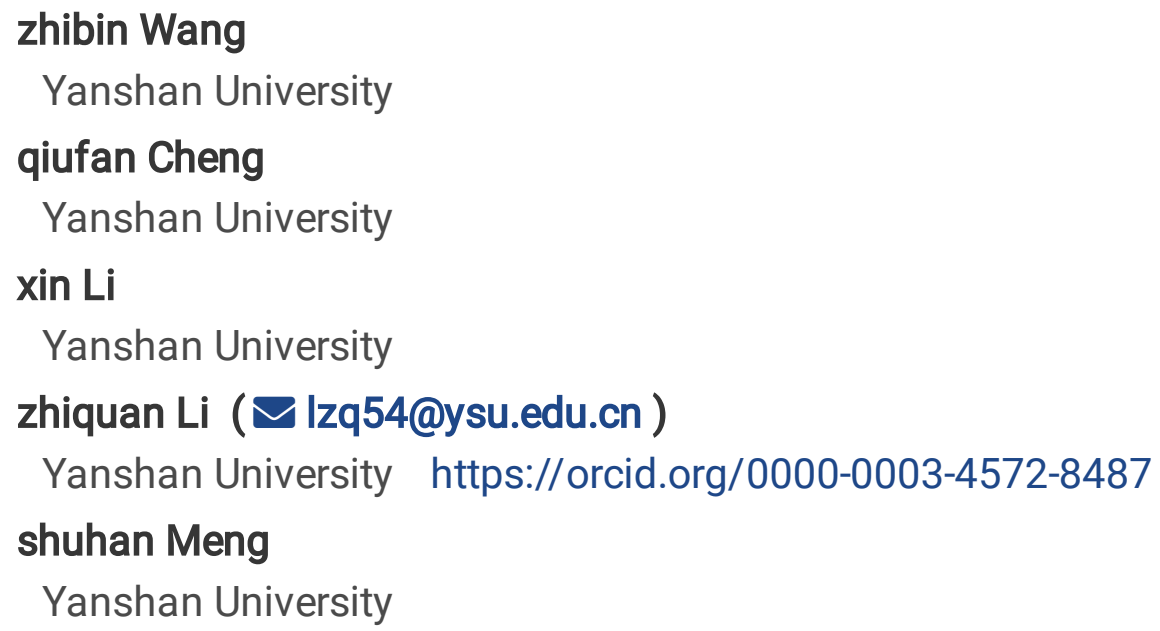

\section{Research Article}

Keywords: Anisotropic epsilon-near-zero (AENZ) metamaterials, Graphene, Nanoglass, Light modulator.

Posted Date: July 7th, 2021

DOI: https://doi.org/10.21203/rs.3.rs-659919/v1

License: (c) (1) This work is licensed under a Creative Commons Attribution 4.0 International License. Read Full License

Version of Record: A version of this preprint was published at Optical and Quantum Electronics on January 4th, 2022. See the published version at https://doi.org/10.1007/s11082-021-03426-5. 


\title{
Research of an optical device based on an anisotropic epsilon-near-zero metamaterial
}

\author{
Zhibin Wang ${ }^{1}$, Qiufan Cheng ${ }^{1}$, Xin LI ${ }^{2}$, Zhiquan Li ${ }^{1},{ }^{*}$, Shuhan Meng ${ }^{1}$ \\ ${ }^{1}$ Institute of Electrical Engineering, Yanshan University, Qinhuangdao 066004, China \\ ${ }^{2}$ Institute of Mathematics and Information Technology, Hebei Normal University of Science and \\ Technology, Qinhuangdao 066004, China \\ *Corresponding author. \\ E-mail address: lzq54@ysu.edu.cn
}

Abstract: In this work, a novel design of an electro-tunable narrow channel based on an anisotropic epsilon-near-zero (AENZ) metamaterial is presented. The ENZ condition can be flexibly tuned by an applied gate voltage. This permittivity-tunable channel is composed of alternating layers of graphene and nanoglass with a thickness of $3 \mathrm{~nm}$. Additionally, this channel is utilized in a dual output light modulator to expand its application. Numerical analysis results show that the maximum transmittance of incident light can reach $96.7 \%$, and the extinction ratio (ER) of the device is $14.8 \mathrm{~dB}$ when the gate voltage is added to $4.96 \mathrm{~V}$ at the near-infrared wavelength based on the finite element method (FEM). This ultracompact optical device may open a new realm in highly integrated photonic circuits, especially on the nano-chips.

Keyword: Anisotropic epsilon-near-zero (AENZ) metamaterials; Graphene; Nanoglass; Light modulator.

\section{Introduction}

Recently, artificial electromagnetic (EM) metamaterials have attracted widespread attention all over the world. Researchers created these materials that do not exist in nature by designing almost arbitrary effective permeability and permittivity. Moreover, these metamaterials have unique optical properties of achieving abnormal regulation of light. The zero-index materials (ZIM), as a specific type of this metamaterials, also have become a popular material in scientific research. They are divided into epsilon-near-zero (ENZ) metamaterials, mu-near-zero (MNZ) metamaterials, and matched impedance zero-index metamaterials (MIZIM). Because the refractive index of these metamaterials tends to zero, there will be a constant phase advance when the EM wave goes through them. At the same time, there will be other exciting phenomena such as the ability to squeeze and tunnel [1], cloaking [2,3] and enhanced coupling [4], etc. All the extraordinary optical phenomenon in the ZIMs may guide potential applications on ultra-energy-efficient, all-optical switching devices for future optical communication and computation. 
The zero-index material was firstly proposed by Pendry [5] and verified by experiments in 2007[6]. Up to now, there are many methods to obtain and regulate zero-index materials. For example, Edwards et al. [7] realized the effect of zero refractive index by using a microwave waveguide setup. Because the EM waves propagate in different modes in metal waveguides and each mode corresponds to a cut-off frequency, the propagation constant will be close to zero when the frequency of the incident wave is precisely at the cut-off frequency for a specific mode. In 2013, Maas, R. et al. [8] found that a zero-index material can be achieved by a multi-layers materteral with alternating positive and negative dielectric according to equivalent medium theory. The common one is that silver can be regarded as a material with a negative dielectric constant in the visible spectral range, while silicon is a dielectric with a positive one. Once they were stacked, the two materials construct a zero refractive index material in the visible range. In addition, scholars also have found that the embedded defects in the zero-index material will affect the reflection and transmission of light. $\mathrm{Xu}$, Y. et al. [9] demonstrated the transverse magnetic (TM) wave transmission of cylindrical defects in the zero-index materials, Wu, Y. et al. [10] studied the rectangular ones, and Huang, Y. et al. [11] displayed the triangular ones, respectively. Results showed that the transmittance of the incident TM wave was influenced by the sizes, quantities, and the dielectric constant of defects. However, it is not easy to control those variables once these defects are embedded in the zero-index material.

Nanoglass, as a lower permittivity material [12], has excellent optical properties. It will not be affected by temperature and has a higher light response speed [13]. Furthermore, it has a giant optical nonlinearity under stable light conditions [14]. Graphene is a two-dimensional material favored by many scholars in optoelectronic research fields over the years. Various applications and devices using graphene have been obtained, including antennas [15], waveguides [16], and switches [17]. Inspired by the previous work [8,21], a metamaterial constructed by alternative layers of graphene and nanoglass is presented due to the electrically tunable property of graphene.

Here, an epsilon-near-zero material channel can be obtained only if the permittivity of the proposed structure is tuned to zero value, which results in a low power dissipation when the incident waves pass through this ultracompact channel. Compared to defects structure, this waveguide structure is more available to realize light modulation which only required a tunable voltage to achieve the tunneling effect. Besides, a light modulator has been illustrated in our simulation demonstrating the output light of two output ports can be arbitrarily selected. 


\section{Theoretical analysis and physical modeling}

\section{1 Searching for epsilon-near-zero point in graphene-nanoglass structure}

In order to design a tunable optical device based on graphene-nanoglass metamaterial, we should first verify the optical properties of the proposed materials. The optical properties of graphene are mainly affected by the electrical conductivity and equivalent permittivity. Hence, tuning the optical property of graphene by changing the gate voltage has been widely used. According to Kubo formulas [18], graphene's conductivity comprises two parts: the intraband and the interband.

$$
\begin{gathered}
\sigma=\sigma_{\text {intra }}+\sigma_{\text {inter }} \\
\sigma_{\text {int } r a}=\frac{-i e^{2}}{\pi \mathrm{h}^{2}\left(\omega+i 2 \tau^{-1}\right)}\left[\int_{0}^{\infty} \varepsilon\left(\frac{\partial f_{d}(\varepsilon)}{\partial \varepsilon}-\frac{\partial f_{d}(-\varepsilon)}{\partial \varepsilon}\right) d \varepsilon\right] \\
\sigma_{\text {inter }}=\frac{-i e^{2}\left(\varpi+i 2 \tau^{-1}\right)}{\pi \mathrm{h}^{2}}\left[\int_{0}^{\infty} \varepsilon\left(\frac{f_{d}(-\varepsilon)-f_{d}(\varepsilon)}{\left(\omega+i 2 \tau^{-1}\right)^{2}-4(\varepsilon / \mathrm{h})^{2}} d \varepsilon\right]\right.
\end{gathered}
$$

where $e$ represents elementary charge $\left(e=1.6 \times 10^{-19} \mathrm{C}\right), \omega$ is the angular frequency of the incident wave, $\mathrm{h}$ is the reduced Planck constant ( $\mathrm{h}=h / 2 \pi, h=6.62 \times 10^{-34} \mathrm{~J}$ s is the Planck constant), $f_{d}(\varepsilon)$ is the Fermi-Dirac distribution, and $\tau$ represents the relaxation time which is closely related to the carrier mobility $\mu$ and the chemical potential $\mu_{c}$.

$$
\begin{gathered}
f_{d}=\left(e^{\left(\varepsilon-\mu_{c}\right) / k_{B} T}+1\right)^{-1} \\
\tau=\frac{\mu \mu_{c}}{e \nu_{F}}
\end{gathered}
$$

where $k_{B}$ is the Boltzmann constant, $T=300 \mathrm{~K}$ is the Kelvin temperature $\left(k_{B} \cdot T=0.026 \mathrm{eV}\right), \mu$ is set to $1000 \mathrm{~cm}^{2} /(\mathrm{Vs})$, and $v_{F}=1 \times 10^{-6} \mathrm{~m} \cdot \mathrm{s}^{-1}$ is the Fermi velocity.

Because the sheet graphene is treated as an extremely thin film, the relationship between the surface permittivity (along the $\mathrm{x}$ - and z-directions) of graphene can be expressed as:

$$
\varepsilon_{g x x}=\varepsilon_{g z z}=1+i \frac{\sigma}{\omega \varepsilon_{0} \Delta}=1-\frac{\operatorname{Im}(\sigma)}{\omega \varepsilon_{0} \Delta}+i \frac{\operatorname{Re}(\sigma)}{\omega \varepsilon_{0} \Delta}
$$

where $\varepsilon_{0}$ is the dielectric constant in vacuum, $\Delta$ represents the thickness of graphene, and we set the thickness of graphene to be $0.6 \mathrm{~nm}$ in all the simulations. Considering the normal electric field cannot excite any current in the y-direction, so the normal component of the graphene's permittivity should be 1. Based on formula (6), we can get the equivalent permittivity of graphene is related to its conductivity and angular frequency. Furthermore, the conductivity will be affected by $\mu_{c}$ which can 
be controlled by an applied voltage. According to the equivalent medium theory, the permittivity tensor of this structure can be obtained from Ref. [18]:

$$
\begin{gathered}
\varepsilon_{\mathrm{xx}}=\varepsilon_{z z}=f_{g} \varepsilon_{g x x}+\left(1-f_{g}\right) \varepsilon_{d} \\
\varepsilon_{\mathrm{yy}}=\left(\frac{f_{g}}{\varepsilon_{g \mathrm{y}}}+\frac{1-f_{g}}{\varepsilon_{d}}\right)^{-1} \\
\mu_{\mathrm{c}}=\mathrm{h} \nu_{F} \sqrt{\pi \eta_{0} \mid V_{0}+V_{g}} \mid
\end{gathered}
$$

where $f_{g}=0.2$ is the filling factor of graphene, $\varepsilon_{d}=1.3$ is the permittivity of nanoglass [19], $\eta_{0}=$ $5.65 \times 10^{16} \mathrm{~m}^{-2} \mathrm{~V}^{-1}, V_{0}$ is the voltage offset caused by the natural doping [20], and $V_{g}$ stands for the applied voltage. Based on the formula (8), we can get the vertical direction effective permittivity of the metamaterial is a constant as 1.226 . According to formula (7), the metamaterial's permittivity tensor along the $\mathrm{x}$ and $\mathrm{z}$-direction can be tuned by the chemical potential $\mu_{c}$ or the incident wavelength. For a given wavelength such as $1550 \mathrm{~nm}$, there is an identical chemical potential $\mu_{c}$ corresponding to the ENZ point. All the simulations were investigated by using the commercial software COMSOL Multiphysics based on FEM.

\section{2 Numerical simulation of the designed structure}

Figure 1(a) depicts a zigzag waveguide structure with two parallel $\mathrm{Si}_{3} \mathrm{~N}_{4}$ waveguides and a graphene-nanoglass metamaterial channel. The channel connects the two parallel Si3N4 waveguides. The whole structure is sealed by perfect electric conductor (PEC) walls. The incident wave is input from the left port and output from the right one. The metamaterial is composed of alternating layers of graphene and nanoglass, as shown in the inset of Figure 1(b). The period thickness of the structure is $3 \mathrm{~nm}$ with a $0.6 \mathrm{~nm}$ graphene sheet and a $2.4 \mathrm{~nm}$ nanolass layer. The widths of the two $\mathrm{Si}_{3} \mathrm{~N}_{4}$ channels are set as $a_{1}=a_{2}=330 n m$ whose permittivity is 2.1 . We assume that our structure is surrounded by air with permittivity of 1 , and the boundaries were set as perfect boundary conditions, which can be seen in Figure 1(c).

$$
T=\frac{2 a_{1}}{\left(a_{1}+a_{2}\right) \cos \left(k_{x} d\right)-i\left(\varepsilon_{y y} \frac{a_{1} a_{2}}{a_{3}}+u_{r} a_{3}\right) k_{0} d \sin \left(k_{x} d\right)}
$$

where $k_{x}=\omega \sqrt{\varepsilon_{0} \varepsilon_{y y} \mu_{0} \mu_{r}}$ is the x-direction vector of the wave, and $\mu_{r}=1$ is the relative permeability of the ENZ metamaterial [21]. 


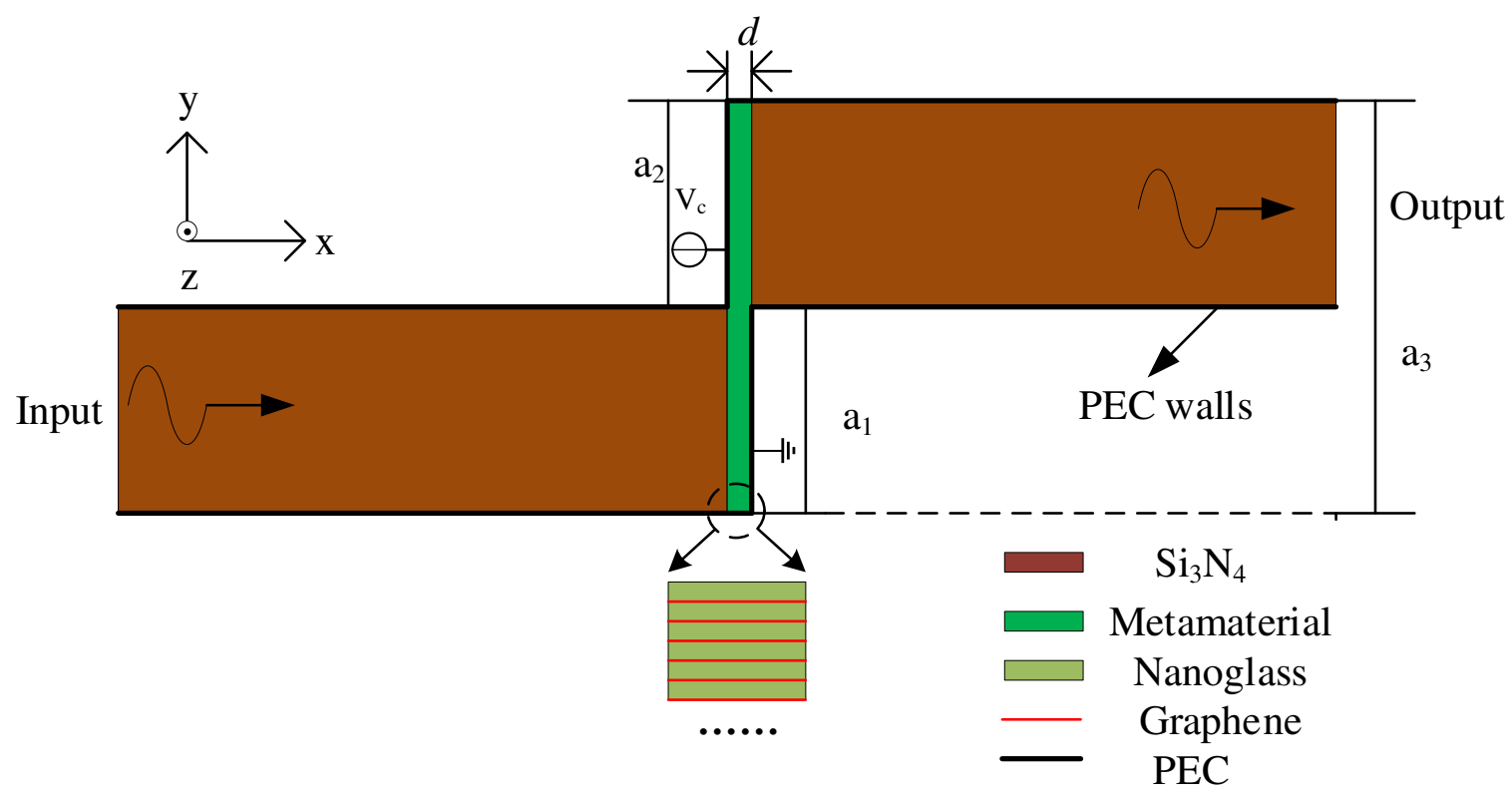

(a)

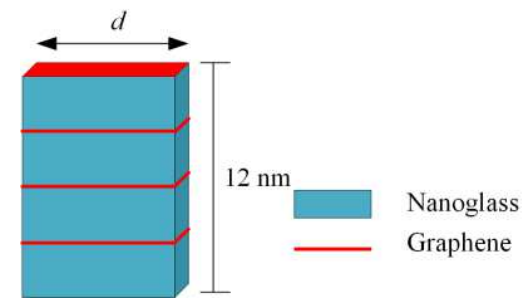

(b)

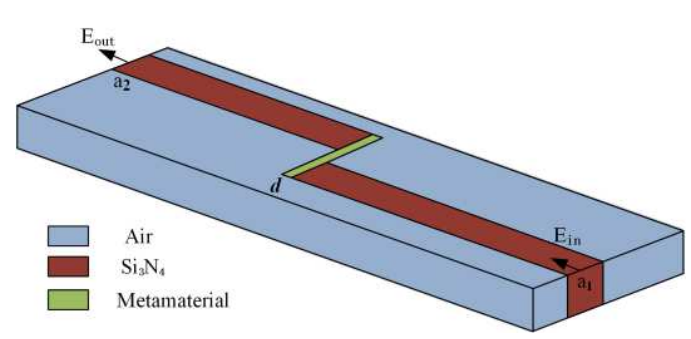

(c)

Fig. 1(a). The sectional view of the waveguide structure together with the construction of the metamaterial. The incident wave is input from the left port. It is squeezed and tunneled through the metamaterial channel and output from the right port. (b). A $12 \mathrm{~nm}$ thickness of the metamaterial consisted of graphene sheets and nanoglass layers. (c). Sketch of the $\mathrm{Air} / \mathrm{Si}_{3} \mathrm{~N}_{4} /$ metamaterial structure.

Since the width of the $\mathrm{Si}_{3} \mathrm{~N}_{4}$ waveguides is much smaller than the incident wavelength of $1550 \mathrm{~nm}$, only the fundamental transverse magnetic mode $\left(\mathrm{TM}_{0}\right)$ can exist in our structure. The length of the metamaterial is set to be $\mathrm{d}=15 \mathrm{~nm}$ according to formula (10), which can achieve a large extinction ratio and an acceptable insertion loss [21].

\section{Results and analysis}

Based on the above analysis, we can get the relationship between the equivalent permittivity and the chemical potential of graphene $\mu_{c}$. Figure 2(a) illustrates the horizontal permittivity $\varepsilon_{\mathrm{xx}}$ of the metamaterial as a function of the chemical potential of graphene $\mu_{c}$ for a specific incident wavelength $\lambda=1550 \mathrm{~nm} . \operatorname{Real}\left(\varepsilon_{\mathrm{xx} 1}\right)$ represents the real permittivity of graphene-nanoglass structure (G-N) and $\operatorname{real}\left(\varepsilon_{\mathrm{xx} 2}\right)$ shows the previous one of graphene-silica structure (G-S) by contrast. The real part $\varepsilon_{\mathrm{xx}}$ is changed from a positive value to a negative one demonstrating that the real part of permittivity is nearly equal to zero for a fixed wavelength. Owing to the chemical potential is higher than the half photon energy, the imaginary part of permittivity is always a small value when $\mu_{c}>0.41 \mathrm{eV}$. 

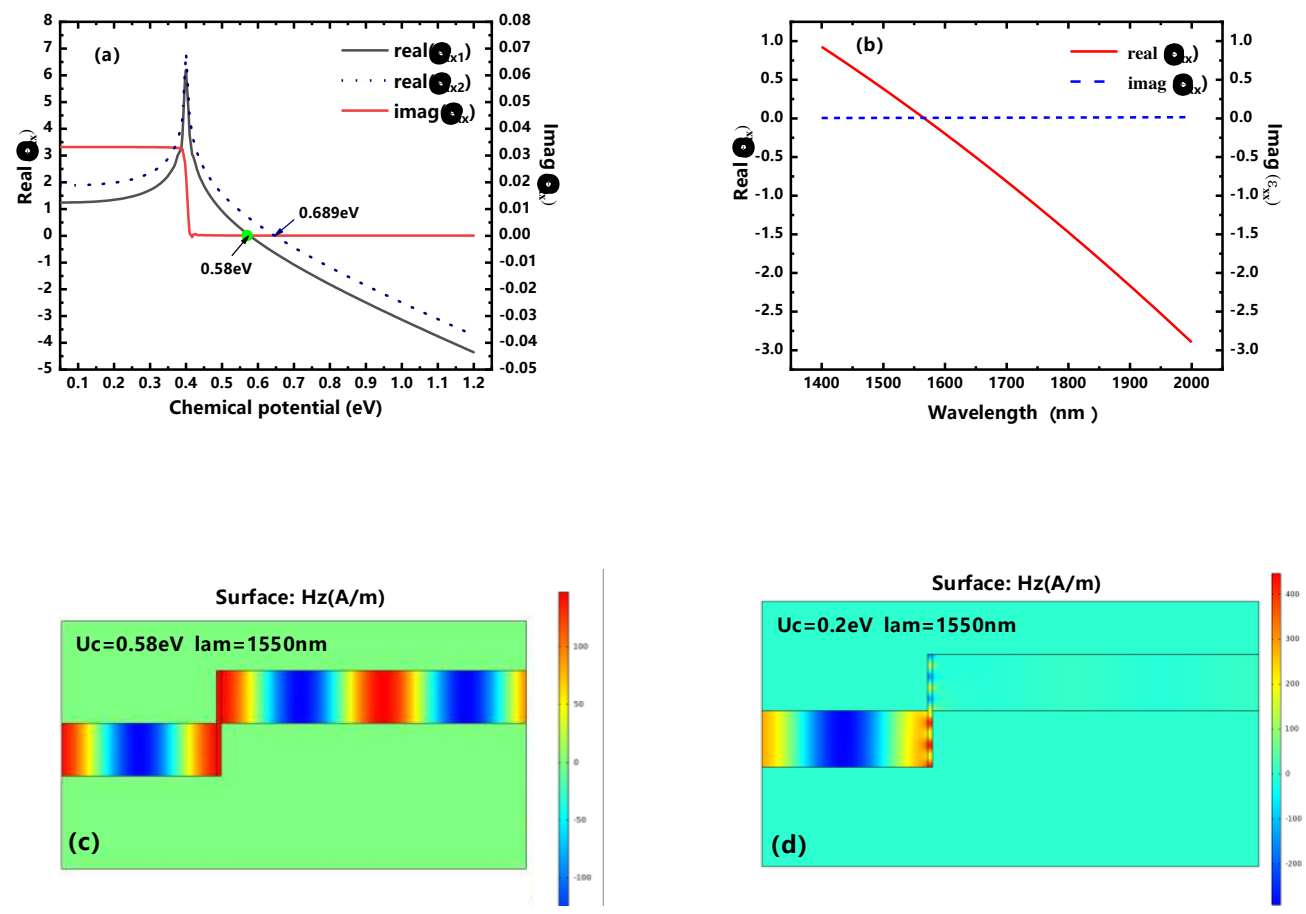

Fig. 2(a) Function of the real and imaginary parts of effective permittivity of the metamaterial in the horizontal direction and the chemical potential of graphene for a fixed wavelength $\lambda=1550 \mathrm{~nm}$. The dashed line in the picture represents the previous work of graphene and silica structure. (b) The relationship between the horizontal permittivity of the metamaterial and various wavelengths of the incident wave for $\mu_{c}=0.58 \mathrm{eV}$. (c) and (d) The magnetic field distribution inside the modulator in z-direction for the $\mathrm{ON}$ and $\mathrm{OFF}$ states, respectively.

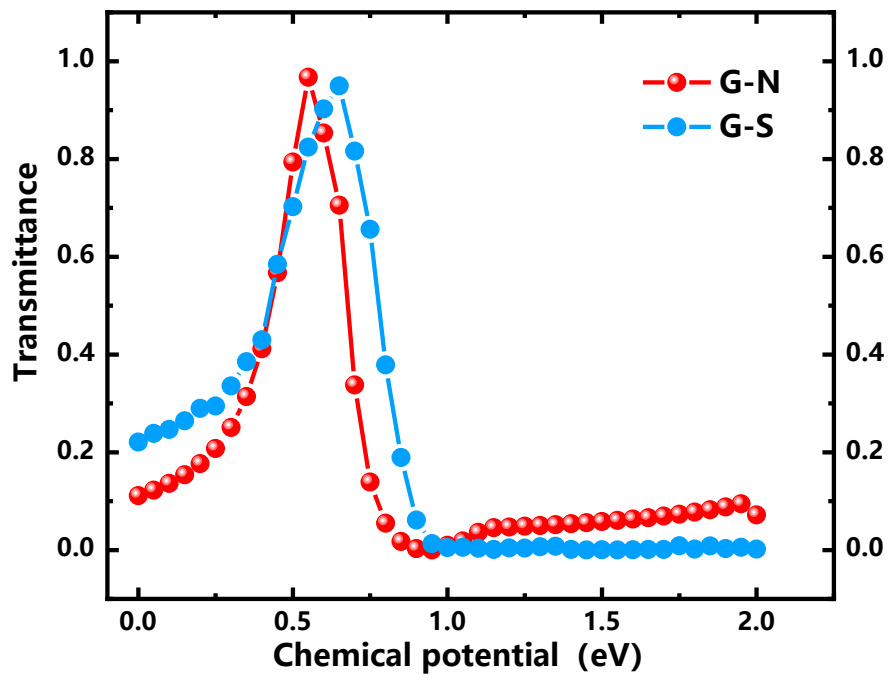

Fig. 3. The transmittance as a function of the chemical potential in the metamaterial for the incident wavelength is $1550 \mathrm{~nm}$.

The results of the simulation are in line with the theoretical predictions. The anisotropic epsilon-near-zero metamaterial can be realized when $\mu_{c}=0.58 \mathrm{eV}$. Apparently, the ENZ point in our work is around $\mu_{c}=0.58 \mathrm{eV}$ for $\lambda=1550 \mathrm{~nm}$, which is lower than previous work for $\mu_{c}=0.689 \mathrm{eV}$ [21]. That means our work only needs a lower applied voltage to arrive at ENZ condition. Figure 2(b) shows that the permittivity of metamaterial is a function of the wavelength of the incident light for a fixed chemical potential $\mu_{c}=0.58 \mathrm{eV}$. The horizontal permittivity of the metamaterial $\varepsilon_{\mathrm{xx}}$ is 
$-0.02517+0.0083 \mathrm{i}$ when the wavelength is $1550 \mathrm{~nm}$, which is equal to zero value to achieve the squeezing and tunneling effect. The distributions of the transverse magnetic field along z-direction for ENZ point of $\mu_{c}=0.58 \mathrm{eV}$ and non-ENZ point of $\mu_{c}=0.2 \mathrm{eV}$ are shown in Figures 2(c) and (d). The light can pass through the structure at ENZ point and get blocked at non-ENZ point, which corresponds to the $\mathrm{ON}$ and OFF states, respectively. Moreover, the transmittance of incident light is shown in Figure 3. The blue line represents the result of graphene-silica structure $(\mathrm{G}-\mathrm{S})$ in previous work, and the red line shows the one of graphene-nanoglass structure $(\mathrm{G}-\mathrm{N})$ in our work. Considering the loss of the ENZ metamaterial, the permittivity of the graphene-silica metamaterial ( $\left.\varepsilon_{\mathrm{xx}}=-0.02949+0.007 \mathrm{i}\right)$ is utilized in the simulation by contrast in Figure 3.The transmission coefficients of the G-N metamaterial for $\mu_{c}=0.58 \mathrm{eV}$ ( $\varepsilon_{\mathrm{xx}}=-0.02547+0.083 \mathrm{i}$ ) is $96.7 \%$, whose maximum transmittance is higher than the previous work of $94.9 \%$ for $\mu_{c}=0.689 \mathrm{eV}[22]$. Additionally, the expression of extinction ratio is shown in equation (11):

$$
\mathrm{ER}=10 \lg \left(\frac{T_{\text {max }}}{T_{\text {min }}}\right)
$$

The maximum and minimum values of the EM field energy ( $\mathrm{Hz}$ at the output of the optical device) are represented by $T_{\max }$ and $T$ min, respectively. It is found that $\mu_{c}=0.2 \mathrm{eV}$ is set to the OFF state $\left(T_{\min }=0.031 \mathrm{~dB}\right)$ and $\mu_{c}=0.58 \mathrm{eV}$ is responded to the $\mathrm{ON}$ state $(T \max =0.935 \mathrm{~dB})$, so the extinction ratio (ER) is obtained as $14.8 \mathrm{~dB}$ according to the formula (11).

Besides, we use this structure to design a dual output light modulator shown in Figure 4(a). The whole waveguide is sealed by perfect electric conductor (PEC) walls, which were used to prevent light energy from leaking out of the ENZ channel. The size of this channel is the same as we proposed before $\left(a_{1}=a_{2}=330 \mathrm{~nm}\right)$, and $\mathrm{a}_{3}$ is set to $165 \mathrm{~nm}$ to reduce the loss of light. The light is input from the bottom port and output from two ports at the top. We assume that this structure is surrounded by air with permittivity of 1 , and the boundaries of the air layer were set as scattering boundary conditions. The wavelength of incident light is $1550 \mathrm{~nm}$. Figure 4(b) and (c) demonstrate the magnetic field distribution inside the light modulator in the z-direction. Thus, this optical device is more flexible than the structure proposed in Ref. [22].

When the $\mu_{c}=0.58 \mathrm{eV}$ arrives at the ENZ point, the result shows that the incident light can pass the two ports at the top. Otherwise, the light will be confined at the input port. Furthermore, this light modulator can make the wave pass the two output ports, respectively, regulating the light freely. In the meanwhile, the energy of one output is demonstrated in Figure 5. The maximum energy of one output can be achieved at $31 \%$ when $\mu_{c}$ is enlarged to $0.58 \mathrm{eV}$. It shows that the proposed structure will take advantage of more opportunities for photonic devices. 

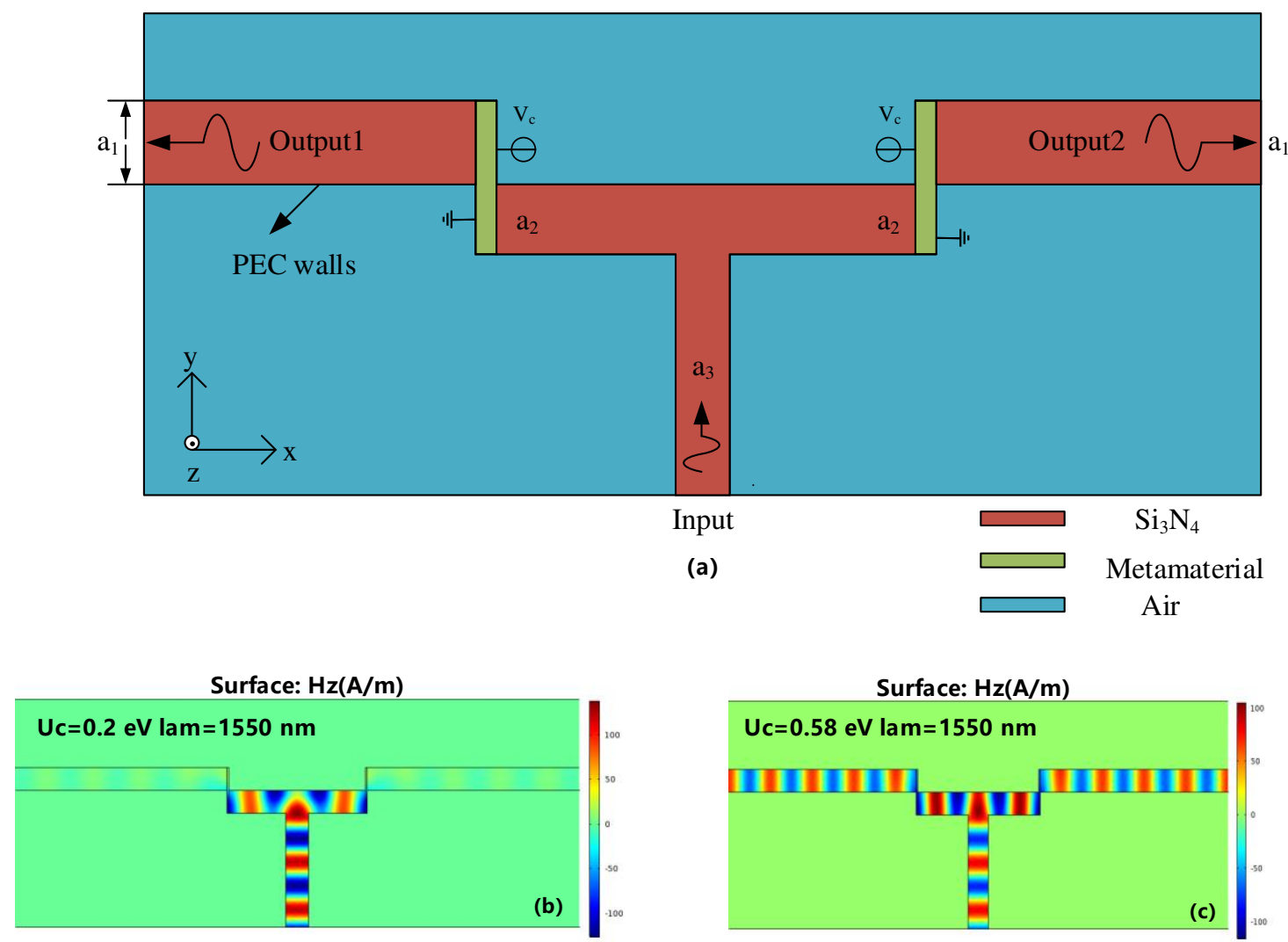

Surface: $\mathrm{Hz}(\mathrm{A} / \mathrm{m})$

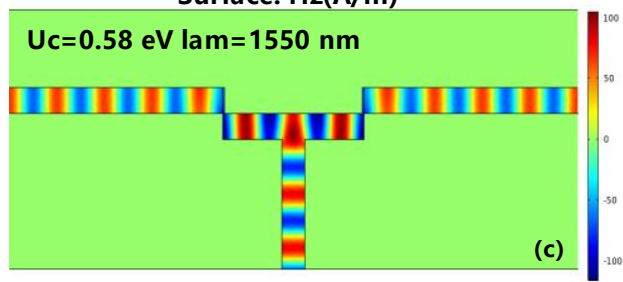

Fig. 4(a) Schematic of the proposed dual output light modulator. (b) and (c) Magnetic field distributions along the z-direction (Hz) for $\mu_{c}=0.2 \mathrm{eV}$ and $\mu_{c}=0.58 \mathrm{eV}$ respectively.

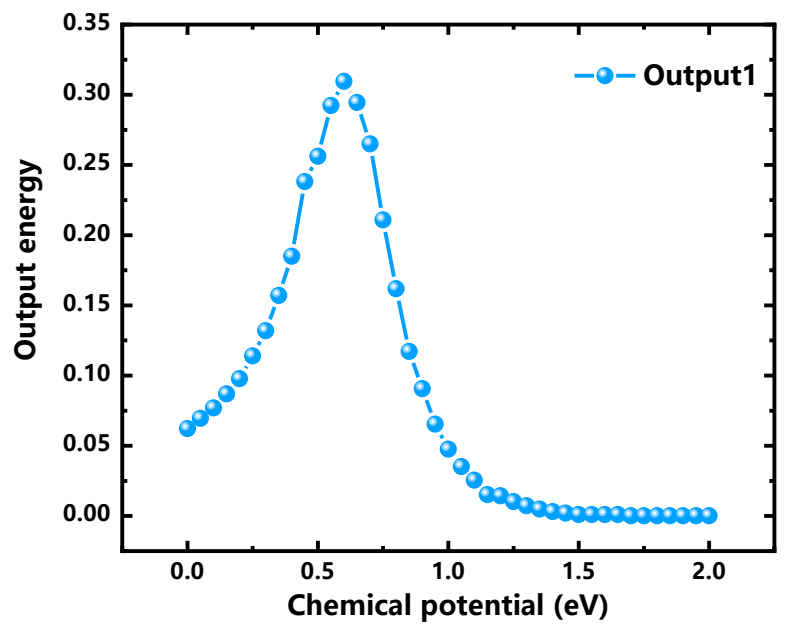

Fig. 5. The output energy of the left output port under different $\mu_{c}$

As a result, the transmittance at the ENZ point adding a lower gate voltage is $96.7 \%$ in our work, which is better than $94.9 \%$ of previous work. Furthermore, a more flexible dual output light modulator on the port selection aspect is proposed, whose maximum energy can be reached about $62 \%$ of the incident wave.

\section{Conclusion}

In summary, the anisotropic epsilon-near-zero metamaterial channel whose permittivity can be 
tuned to near zero is designed in this paper. The variation of the permittivity is influenced by different chemical potential $\mu_{c}$ and different wavelengths. For a fixed wavelength, the channel can be regulated by an applied voltage. The optical energy of the incident light can be tunneled and regulated due to the narrow channel in the light modulator. All the results are essential to optical interconnects and optoelectronic systems, which have potential importance to guide in highly integrated photonic circuits reducing the size of on-chip optical devices.

\section{Declarations}

Funding

This work was supported by the Natural Science Foundation of Hebei Province grant (No: F2017203316) in China, the Youth Fund Project of Hebei Provincial Department of Education (No: QN2019061) in China.

\section{Conflicts of interest/Competing interests}

The authors declared that they have no conflicts of interest in this work.

\section{Availability of data and material}

The data sets used or analyzed during the current study are available from the corresponding author on reasonable request.

Code availability

Not applicable.

Authors' contributions

Zhibin Wang, Qiufan Cheng, Xin Li contributed to the conception of the study;

Zhibin Wang, Qiufan Cheng, Zhiquan Li contributed to analysis and manuscript preparation;

Qiufan Cheng and Shuhan Meng performed the data analyses and wrote the manuscript.

\section{Ethics approval}

Not applicable.

\section{Consent to participate}

Informed consent was obtained from all individual participants included in the study.

\section{Consent for publication}

The participant has consented to the submission of the case report to the journal.

\section{References}

[1] Silveirinha M, Engheta N. Tunneling of Electromagnetic Energy through Subwavelength Channels and Bends using Epsilon-Near-Zero Materials[J]. Physical Review Letters,2006, 97(15):157403.

[2] Kundtz N, Smith D R. Extreme-angle broadband metamaterial lens. Nat Mater 9:129-132[J]. Nature Materials.

[3] Papasimakis N, Thongrattanasiri S, Zheludev N I, et al. The magnetic response of graphene split-ring metamaterials[J]. Light Science \& Applications, 2013, 2(7): e78.

[4] Ourir A, Maurel A, Pagneux V. Tunneling of electromagnetic energy in multiple connected leads using -near-zero materials[J]. Optics Letters, 2013, 38(12):2092-2094.

[5] Pendry J B. Negative Refraction Makes a Perfect Lens[J]. Physical Review Letters,2000, 85(18):3966-3969. 
[6] Wood B, Pendry J B. Metamaterials at zero frequency[J]. Phys Condens Matter,2007, 19(7):076208.

[7] Edwards, B., Alu, A., Young, M.E., Silveirinha, M. and Engheta, N., Experimental verification of epsilon-near-zero metamaterial coupling and energy squeezing using a microwave waveguide, Phys. Rev. Lett., 100, 033903, (2008).

[8] Maas, R., Parsons, J., Engheta, N. and Polman A, Experimental realization of an epsilon-near-zero metamaterial at visible wavelengths. Nat. Photon., 7, 907-912, (2013).

[9] Xu, Y. and Chen, H, Total reflection and transmission by epsilon-near-zero metamaterials with defects, Appl. Phys. Lett., 98, 113501 (2011).

[10] Wu, Y., and Li, J., Total reflection and cloaking by zero-index metamaterials loaded with rectangular dielectric defects, Appl. Phys. Lett., 101, 4184 (2013).

[11] Huang, Y., Li, J. Total reflection and cloaking by triangular defects embedded in zero-index metamaterials. Adv. Appl. Math. Mech., 7, 135-144 (2015).

[12] Reynard J P, Verove C, Sabouret E, et al. Integration of fluorine-doped silicon oxide in copper pilot line for 0.12- $\mu \mathrm{m}$ technology[J]. Microelectronic Engineering, 2002, 60(1):113-118.

[13] Danilov D, Hahn H, Gleiter H, et al. Mechanisms of Nanoglass Ultrastability[J]. ACS Nano, 2016, 10(3):3241-3247.

[14] Wu K, Y Meng, Xu J, et al. Novel Fe-based Nanoglass as Efficient Noble-Metal-Free Electrocatalyst for Alkaline Hydrogen Evolution Reaction[J]. Scripta Materialia, 2020, 188:135-139.

[15] Xu Z, Zhu J, Xu W, et al. Novel graphene enhancement nanolaser based on hybrid plasmonic waveguides at optical communication wavelength[J]. Chinese Physics B, 2018, v.27(08):46-51.

[16] Nair, R. R, Blake, P, Grigorenko, A. N, et al. Fine Structure Constant Defines Visual Transparency of Graphene[J]. Science, 320(5881):1308-1308.

[17] Lu Z. Nanoscale electro-optic modulators based on graphene-slot[J]. Journal of the Optical Society of America B, 2012, 29(6):1490-1496.

[18] Stauber T, Peres N, Geim A. Optical conductivity of graphene in the visible region of the spectrum. Physical Review B.2008; 78:085432.

[19] Bofeng, Zhu, Guobin, et al. Nanoscale dielectric-graphene-dielectric tunable infrared waveguide with ultrahigh refractive indices[J]. Optics Express, 2013.

[20] Liu M, Yin X, Ulin-Avila E, et al. A graphene-based broadband optical modulator[J]. Nature, 2011, 474(7349):64-67.

[21] Yang L, Hu T, Shen A, et al. Ultracompact optical modulator based on graphene-silica metamaterial[J]. Optics Letters, 2014, 39(7):1909-12.

[22] Liu, et al. Numerical investigation of a multi-functional optical device based on graphene-silica metamaterial. [J]. Journal of Modern Optics, 2016. 